\title{
High-Frequency Impulse Therapy for Treatment of Chronic Back Pain: A Multicenter Randomized Controlled Pilot Study
}

\author{
Kasra Amirdelfan' \\ Mindy Hong (D) ${ }^{2}$ \\ Bobby Tay ${ }^{3}$ \\ Surekha Reddy ${ }^{4}$ \\ Vinay Reddy ${ }^{4}$ \\ Michael Yang ${ }^{5}$ \\ Krishn Khanna ${ }^{3}$ \\ Prasad Shirvalkar ${ }^{6}$ \\ Christopher Abrecht ${ }^{7}$ \\ Amitabh Gulati $\mathbb{1 D}^{8}$ \\ 'IPM Medical Group, Walnut Creek, CA, \\ USA; ${ }^{2}$ Hinge Health, San Francisco, CA, \\ USA; ${ }^{3}$ Department of Orthopaedic \\ Surgery, University of California San \\ Francisco, San Francisco, CA, USA; \\ ${ }^{4}$ Spine and Nerve Diagnostic Center, \\ Roseville, CA, USA; ${ }^{5}$ Summit Pain \\ Alliance, Santa Rosa, CA, USA; ${ }^{6}$ UCSF \\ Weill Institute of Neurosciences \\ University of California San Francisco, \\ San Francisco, CA, USA; ${ }^{7}$ Department of \\ Anesthesia and Perioperative Care, \\ University of California San Francisco, \\ San Francisco, CA, USA; ${ }^{8}$ Memorial Sloan \\ Kettering Cancer Center, New York, \\ NY, USA
}

Correspondence: Amitabh Gulati Memorial Sloan Kettering Cancer Center, 1275 York Avenue, New York, NY, 10065, USA

Tel + I 212 639-2000

Email amitabhgulati@gmail.com
Purpose: This study aims to examine high-frequency impulse therapy (HFIT) impact on pain and function among patients undergoing care for chronic low back pain (CLBP).

Methods: A pilot randomized-controlled trial of HFIT system versus sham was conducted across 5 orthopedic and pain center sites in California, USA. Thirty-six patients seeking clinical care for CLBP were randomized. Primary outcome was function measured by the Six Minute Walk Test (6MWT). Secondary outcomes were function (Timed Up and Go [TUG] and Oswestry Disability Index [ODI]), pain (Numerical Rating Scale [NRS]), quality of life (Patient Global Impression of Change [PGIC]), and device use. Patients were assessed at baseline and every week for 4 weeks of follow-up. Mann-Whitney $U$-test was used to analyze changes in each outcome. Repeated measures ANOVA was used to assess the effect of treatment over time.

Results: The average age of subjects was $53.9 \pm 15.7$ (mean \pm SD) years, with $12.1 \pm 8.8$ years of chronic low back pain. Patients who received an HFIT device had a significantly higher 6MWT score at weeks 2 [Cohen's $d(95 \% \mathrm{CI}): 0.33(0.02,0.61)], 3[0.32(0.01,0.59)]$ and $4[0.31$ $(0.01,0.60)]$, respectively, as compared to their baseline scores $(\mathrm{p}<0.05)$. Patients in the treatment group had significantly lower TUG scores at week $3[0.30(0.04,0.57)]$ and significantly lower NRS scores at weeks $2[0.34(0.02,0.58)]$ and $4[0.41(0.10,0.67)](p<0.05)$.

Conclusion: A larger-scale RCT can build on the findings of this study to test whether HFIT is effective in reducing pain and improving function in CLBP patients. This study shows encouraging evidence of functional improvement and reduction in pain in subjects who used HFIT. The efficacy and minimally invasive nature of HFIT is anticipated to substantially improve the management of CLBP patients.

Keywords: HFIT, pain, chronic pain, neuromodulation, noninvasive treatment

\section{Introduction}

Chronic low back pain (CLBP) poses significant morbidity for patients, affecting over 76 million people in the United States with total costs related to low back pain (LBP) exceeding $\$ 100$ billion per year. ${ }^{1}$ Existing pharmaceutical treatments, such as opioids, can be effective solutions to CBLP, but tend to cause side effects, including sedation, nausea, constipation and respiratory depression. ${ }^{2}$ Approximately $51 \%$ of patients who live with back pain are unable to tolerate pharmacological pain treatments due to such side effects. ${ }^{3}$ Exercise therapy is a commonly used and moderately effective approach for managing CLBP; ${ }^{4}$ however, it may take several weeks to experience pain relief. Newer treatments in neuromodulation for refractory CLBP include novel devices in spinal cord and 
peripheral nerve stimulation. ${ }^{5}$ While these technologies have improved options for pain control, they require surgical implantation, which increases risks to patients and costs to payors. As a result, there continues to be a great need for an effective, drug-free, non-implanted treatment for CLBP.

Transcutaneous electrical nerve stimulation (TENS) is a form of electrical stimulation therapy used by pain physicians and physiotherapists to treat both chronic and acute forms of musculoskeletal pain. Studies have determined that while TENS can be effective for temporary treatment of musculoskeletal pain, it has not been demonstrated to be effective for long-term treatment of chronic musculoskeletal pain. ${ }^{6}$ A significant limitation for TENS is the ability to deliver adequate and precise electrical fields through the skin, which acts as a resistor. ${ }^{7}$ Methods to overcome the capacitance of the skin structures include higher energy delivery (increased current or intensity) or higher voltage in order to charge the skin structures, such that subsequent field charges may pass through the skin. Studies have demonstrated that high-frequency forms of electrical stimulation (10 kHz and greater frequencies) are effective for the treatment of chronic and acute pain and are thought to be due to the different mechanism of action of high-frequency neuromodulation. ${ }^{8-10}$ The ultra-high frequency system, known as high-frequency impulse therapy (HFIT), involves using specific waveforms in the 30$150 \mathrm{kHz}$ range to both overcome the resistance of the skin and to deliver a higher frequency than TENS. Studies have also demonstrated that high frequency electrical stimulation can affect the firing of action potentials. ${ }^{11}$

This study describes a pilot study using an HFIT device (Enso, San Francisco, CA) in order to evaluate the impact on pain reduction and increase in function among patients with CLBP. We hypothesize that the cohort randomized to use the HFIT device will have a clinically significant increase in function and a clinically significant decrease in back pain compared to the sham group. This study also aims to examine patient use of the HFIT device versus the sham device.

\section{Methods}

\section{Study Design}

This is a pilot, double-blind, placebo-controlled randomized trial, designed to assess functional and pain outcomes of utilizing an HFIT device in subjects with CLBP due to mechanical back pain, degenerative disc disease, degenerative arthritis or disc compression. Patient recruitment and in-person data collection were performed at 5 clinical sites (orthopedic and pain centers) in California, USA. Patients were evaluated at baseline and then with weekly follow-up visits for 4 weeks. The preliminary, interim 4 -week results from the first 36 patients enrolled in this protocol are presented here. The study was registered on ClinicalTrials.gov, (Identifier NCT03320863), approved by the institutional review boards of University of California San Francisco (UCSF) and conducted in accordance with the Declaration of Helsinki.

\section{Participants}

Subjects were evaluated, consented and enrolled based on the protocol's inclusion and exclusion criterion. Key inclusion criteria were (1) Mechanical or non-specific axial back pain, as diagnosed by a board-certified physician; (2) Baseline pain of $\geq 6.0$ out of 10.0 on the Numerical Rating Scale (NRS); (3) Functional debilitation by pain (e.g. difficulty walking); (4) Minimal radicular symptoms with no effect on functionality, medication or quality of life; (5) Expressing disability $\geq$ $80 \%$ due to LBP (based on Likert scale); and (6) Experiencing chronic pain for $\geq 6$ months. Key exclusion criteria included (1) Not owning or having access to a smartphone; (2) Spinal instability, joint instability, or $\geq$ grade 2 spondylolisthesis with instability; (3) Primary symptoms due to spinal stenosis; (4) Diagnosis of cancer or malignant tumors in the last 5 years and (5) Undergoing surgery to solve pain related to study indication in the past 6 months.

The treatment group was given an HFIT device (Figure 1) and instructed to use the device for one hour or more daily. The control group was provided with a sham HFIT device that displayed LED light animations in order to simulate treatment (without delivering electrical pulses) and instructed to use the device for one hour or more daily. In both groups, patients applied the device themselves and controlled treatment durations and intensities through a smartphone app. The app included a HIPAA compliant chat feature that allowed subjects to ask questions and resolve technical issues (e.g. pad use or placement). A study coordinator also contacted participants in both groups three times per week to provide support and answer questions.

\section{Randomization}

Clinicians at participating clinical sites determined eligibility during the patients' appointments and described the study. Research coordinators at the clinical sites then 


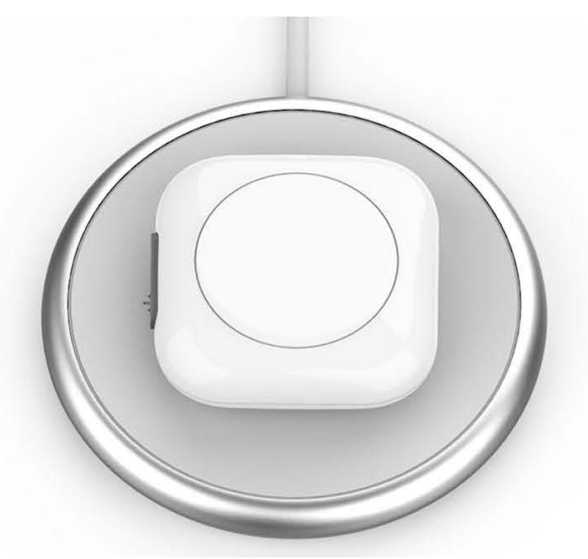

Figure I HFIT Device.

Notes: HFIT device (left), HFIT device worn on model (right).

Abbreviation: HFIT, high-frequency impulse therapy.

conducted the informed consent process. Subjects were randomized 1:1 to receive either an HFIT device or a sham HFIT device to supplement their existing pain regiment. Allocation concealment was ensured using the sequentially numbered opaque sealed envelopes (SNOSE) method. Participants, investigators and staff interacting with patients were all blinded to subject assignments and did not have access to information regarding which patients received a functional device and which patients received a sham device. Participants were told that depending on the treatment they received, the device may or may not provide a sensation. Study site coordinators explained it was possible to deliver high frequency electrical energy across the skin without sensation.

On weeks 1, 2, 3 and 4, patients were prompted by a smartphone app to fill out questionnaires concerning functionality and quality of life. Patients also visited the clinic again to perform follow-up testing for functionality through the Six Minute Walking Test (6MWT) and Timed Up and Go Test (TUG) at weeks 1, 2, 3 and 4. After the 4-week visit, participants were informed of group assignment.

\section{Outcomes}

The primary outcome of the study was 6MWT, utilized to assess functional mobility. 6MWT measures the distance walked (in meters) within 6 minutes, with higher values indicating better outcomes. Secondary outcomes measuring functional mobility included the TUG test, which measures the time it takes (in seconds) to stand, walk 3 meters and return to a seated position, as well as

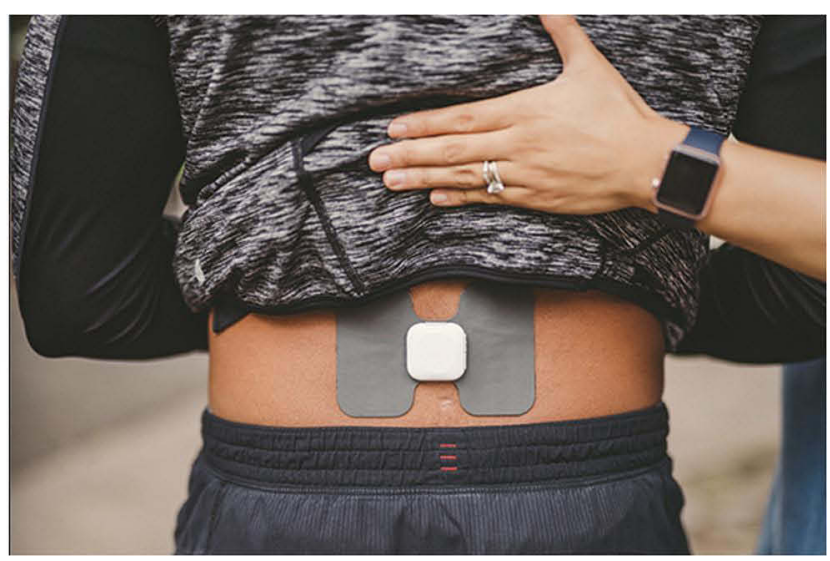

the Oswestry Disability Index (ODI), which ranges from 0 ("no disability") to 50 ("most disability").

The Numerical Rating Scale (NRS) was used to evaluate pain, where scores ranged from 0 ("no pain") to 10 ("worst pain imaginable"). The Patient Global Impression of Change (PGIC) was used to measure the patient's belief about the efficacy of treatment, which ranged from 1 ("no change") to 7 ("a great deal better"). In order to evaluate whether subjects in one group used their device more frequently than another group, device utilization was also measured through the app (in hours per week).

All outcome measures were assessed at predefined study visits (baseline, 1, 2, 3, and 4 weeks). Functionality tests were administered by a clinician at a clinical site. Patient-reported outcome measures were recorded through self-administered questionnaires completed by patients. Data was collected through daily surveys and weekly questionnaires via a smartphone app.

\section{Statistical Analysis}

Because this was a pilot study, formal sample size calculations were not conducted. However, to ensure a reliable estimate in powering a future full-scale study with $90 \%$ statistical power, a minimum of 15 participants per treatment arm is recommended if a moderate effect size between 0.3 and 0.7 is expected. ${ }^{12}$ To ensure the validity of pilot studies, 15 to 20 participants per treatment arm are typically required..$^{13}$ The present study enrolled a total of 36 participants. The results of this pilot study will be used to provide the necessary parameters for a future larger trial.

Baseline demographic and clinical characteristics were presented as proportions or as means with standard 
deviations. To assess improvement, the Mann-Whitney $U$-test was used to compare whether changes in scores from baseline to 4 weeks in the treatment group were greater than the control group, where a one-sided $p<0.05$ was considered statistically significant. Effect sizes (Cohen's $d$ ) were estimated to show the magnitude of the experimental effect. 95\% confidence intervals (CI) around effect size were estimated by bootstrap (using 1000 replications). Longitudinal results were assessed using two-way mixed analysis of variance (ANOVA). Bonferroni correction was used to correct for multiple comparisons. Complete case analysis was used. Analyses were performed using $\mathrm{R}$ version 4.0.5 (R Core Team, Vienna, Austria).

\section{Results}

\section{Population Characteristics}

From April 2017 to September 2018, patients were enrolled and assessed for eligibility with 36 subjects proceeding through baseline evaluations and randomized (17 HFIT therapy, 19 sham; Figure 2). The average age of all randomized subjects was $53.9 \pm 15.7$ (mean \pm SD) years and the average length of chronic back pain was $12.1 \pm 8.8$ years. The average baseline 6MWT, TUG, NRS pain, and ODI scores were $348 \pm 78.7$ meters, $12.9 \pm 2.4$ seconds,
$7.5 \pm 0.9$, and $42.1 \pm 13.8$, respectively. Baseline characteristics for the two groups are shown in Table 1.

\section{Outcomes}

\section{Function}

The study examined patient function over time. Patients who were randomized to HFIT had a significantly greater improvement in 6MWT (primary outcome) than subjects who received the sham device at Week $2(61.46 \pm 48.22$ vs $21.60 \pm 55.53$ meters, $\mathrm{p}=0.024)$ [Cohen's d (95\% CI): 0.33 $(0.02,0.61)]$, Week $3(71.07 \pm 44.50$ vs $42.13 \pm 59.14$ meters, $\mathrm{p}=0.030)[0.32(0.01,0.59)]$ and Week $4(89.57 \pm 54.36 \mathrm{vs}$ $55.53 \pm 66.53$ meters, $\mathrm{p}=0.035)$ [0.31 (0.01, 0.60)], compared to their baseline scores (Table 2).

Additionally, subjects who received the HFIT device also had a significantly greater improvement in TUG than subjects who received the sham device at Week $3(-4.07 \pm$ 2.40 vs $-2.45 \pm 1.94$ seconds, $\mathrm{p}=0.039)$ [0.30 (0.04, $0.57)$ ]. No significant differences were found between the ODI scores of the groups.

\section{Pain}

When examining pain, we found a significantly greater improvement in NRS pain scores in patients who received the HFIT device at Week $2(-3.83 \pm 1.99$ vs $-2.00 \pm 2.30$,

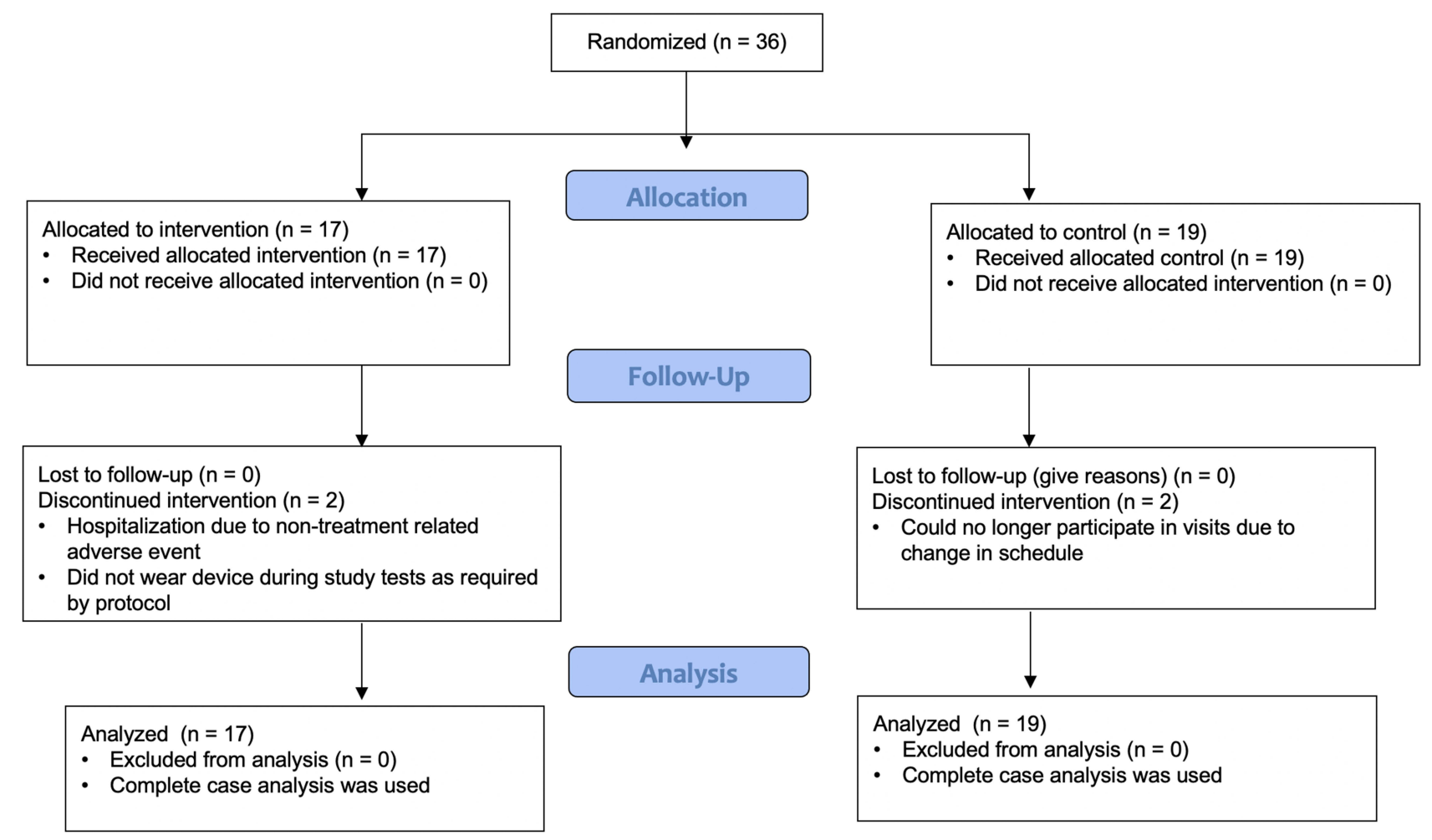

Figure 2 Flowchart. 
Table I Baseline Characteristics

\begin{tabular}{|c|c|c|c|}
\hline & Treatment $(\mathrm{N}=\mid 7)$ & Control $(\mathrm{N}=19)$ & Total $(\mathrm{N}=36)$ \\
\hline \multicolumn{4}{|l|}{ Age (years) } \\
\hline Mean \pm SD & $54.0 \pm 15.7$ & $53.9 \pm 16.2$ & $53.9 \pm 15.7$ \\
\hline Min - Max & $30.0-75.0$ & $26.0-80.0$ & $26.0-80.0$ \\
\hline \multicolumn{4}{|l|}{ Sex - freq (\%) } \\
\hline Male & $3(17.6 \%)$ & $6(31.6 \%)$ & $9(25.0 \%)$ \\
\hline Female & I3 (76.5\%) & $13(68.4 \%)$ & $26(72.2 \%)$ \\
\hline \multicolumn{4}{|l|}{ Race - freq (\%) } \\
\hline White & $13(76.5 \%)$ & I8 (94.7\%) & 31 (86.1\%) \\
\hline Non-white & $4(23.5 \%)$ & I (5.3\%) & $5(13.9 \%)$ \\
\hline \multicolumn{4}{|l|}{ Weight (lbs) } \\
\hline Mean $\pm S D$ & $186 \pm 59.5$ & $182 \pm 39.0$ & $184 \pm 49.1$ \\
\hline \multicolumn{4}{|c|}{ Length of pain (years) } \\
\hline Mean \pm SD & $12.2 \pm 6.72$ & $12.1 \pm 10.0$ & $12.1 \pm 8.80$ \\
\hline \multicolumn{4}{|c|}{ 6MWT (meters) } \\
\hline Mean \pm SD & $337 \pm 93.9$ & $358 \pm 63.1$ & $348 \pm 78.7$ \\
\hline \multicolumn{4}{|l|}{ TUG (seconds) } \\
\hline Mean \pm SD & $13.2 \pm 2.9$ & $12.5 \pm 1.8$ & $12.9 \pm 2.4$ \\
\hline \multicolumn{4}{|l|}{ NRS Pain } \\
\hline Mean \pm SD & $7.5 \pm 0.8$ & $7.5 \pm 1.0$ & $7.5 \pm 0.9$ \\
\hline \multicolumn{4}{|l|}{ ODI } \\
\hline Mean \pm SD & $40.9 \pm 10.2$ & $43.1 \pm 16.5$ & $42.1 \pm 13.8$ \\
\hline \multicolumn{4}{|l|}{ PGIC } \\
\hline Mean \pm SD & $4.3 \pm 0.8$ & $4.2 \pm 0.6$ & $4.2 \pm 0.7$ \\
\hline
\end{tabular}

Abbreviations: SD, standard deviation; 6MWT, Six Minute Walk Test; TUG, Timed Up and Go; NRS, Numerical Rating Scale; ODI, Oswestry Disability Index.

$\mathrm{p}=0.023)[0.34(0.02,0.58)]$ and Week $4(-4.21 \pm 2.49 \mathrm{vs}$

$-1.88 \pm 2.22, \mathrm{p}=0.007)[0.41(0.10,0.67)]$.

\section{Beliefs and Device Usage}

The study also examined beliefs about treatment efficacy and how often devices were used each week. We found that patients assigned to the HFIT device reported a significantly greater improvement in PGIC than subjects who received the sham device at Week $1(0.93 \pm 0.80$ vs $0.29 \pm 0.59, \mathrm{p}=0.006)[0.43(0.12,0.71)]$ and Week 4 $(1.17 \pm 0.94$ vs $0.20 \pm 1.47, \mathrm{p}=0.022)[0.34(0.04,0.62)]$, compared to their baseline scores.

Furthermore, we also found that subjects who were provided with a HFIT device used their device significantly more often than subjects who had received a sham device ( $26.00 \pm 18.86$ vs $19.98 \pm 20.90$ hours per week, $\mathrm{p}=0.010$ ).

In two-way mixed ANOVA for repeated measures, there was not enough evidence to show a statistically significant two-way interaction between treatment and time for the outcomes of interest. There was a significant main effect of time on 6MWT $[\mathrm{F}(2.59,28.5)=21.6, \mathrm{p}<0.001]$, TUG [F $(1.74,19.1)=27.1, \mathrm{p}<0.001]$, NRS pain $[\mathrm{F}(2.72,27.2)=$ 9.50, $\mathrm{p}<0.001]$, ODI $[\mathrm{F}(2.09,25.1)=6.74, \mathrm{p}<0.05]$, and PGIC $[\mathrm{F}(2.11,29.6)=1.19, \mathrm{p}<0.001]$, demonstrating that the mean outcome scores after the HFIT treatment were significantly better than the mean outcome scores before the treatment.

\section{Discussion}

To date, this is the first pilot RCT study to utilize ultrahigh frequency transcutaneous stimulation for the treatment of CLBP. Exploratory analyses showed improved function, pain and device usage outcomes in the HFIT active arm compared to the sham arm as a trend over time (Figure 3). Results indicate a statistical improvement in functional outcomes 6MWT and TUG over time, but not in ODI. Although all are functional outcomes, 6MWT and 
Table 2 Assessment of Outcomes Over Time

\begin{tabular}{|c|c|c|c|}
\hline Measure & Timepoint & Effect Size $(95 \% \mathrm{Cl})$ & p-value \\
\hline \multirow[t]{5}{*}{ 6MWT } & Day I After & $0.03(-0.30,0.30)$ & $p=0.4370$ \\
\hline & Week I & $0.21(-0.11,0.5 I)$ & $p=0.1036$ \\
\hline & Week 2 & $0.33(0.02,0.61)$ & $P=0.0238 *$ \\
\hline & Week 3 & $0.32(0.01,0.59)$ & $P=0.0302 *$ \\
\hline & Week 4 & $0.31(0.01,0.60)$ & $P=0.0350 *$ \\
\hline \multirow[t]{5}{*}{ TUG } & Day I After & $0.18(0.15,0.48)$ & $p=0.1479$ \\
\hline & Week I & $0.28(0.05,0.56)$ & $p=0.0506$ \\
\hline & Week 2 & $0.23(0.11,0.54)$ & $P=0.0908$ \\
\hline & Week 3 & $0.30(0.04,0.57)$ & $\mathrm{P}=0.0393^{*}$ \\
\hline & Week 4 & $0.22(0.12,0.49)$ & $P=0.1024$ \\
\hline \multirow[t]{6}{*}{ NRS Pain } & Day I Before & $0.003(-0.32,0.33)$ & $P=0.5129$ \\
\hline & Day I After & $0.22(-0.53,0.12)$ & $P=0.0971$ \\
\hline & Week I & $0.26(-0.52,0.08)$ & $P=0.0635$ \\
\hline & Week 2 & $0.34(0.02,0.58)$ & $P=0.0227^{*}$ \\
\hline & Week 3 & $0.11(0.25,0.47)$ & $P=0.2524$ \\
\hline & Week 4 & $0.4 \mathrm{I}(0.10,0.67)$ & $P=0.007 I^{*}$ \\
\hline \multirow[t]{4}{*}{ ODI } & Week I & $0.12(-0.22,0.44)$ & $p=0.7607$ \\
\hline & Week 2 & $0.02(-0.36,0.32)$ & $P=0.4623$ \\
\hline & Week 3 & $0.09(-0.26,0.39)$ & $P=0.7016$ \\
\hline & Week 4 & $0.22(0.11,0.49)$ & $p=0.1011$ \\
\hline \multirow[t]{4}{*}{ PGIC } & Week I & $0.43(0.12,0.71)$ & $P=0.0057 *$ \\
\hline & Week 2 & $0.26(-0.06,0.55)$ & $P=0.0591$ \\
\hline & Week 3 & $0.19(-0.12,0.49)$ & $P=0.1302$ \\
\hline & Week 4 & $0.34(0.04,0.62)$ & $P=0.0223 *$ \\
\hline
\end{tabular}

Notes: Mann-Whitney U-test of timepoint versus first timepoint. ${ }^{p}<0.05$; Cohen's metric for effect sizes: 0.2 - small, 0.5 - medium, 0.8 - large.

Abbreviations: 6MWT, Six Minute Walk Test; TUG, Timed Up and Go; NRS, Numerical Rating Scale; ODI, Oswestry Disability Index; PGIC, Patient Global Impression of Change.

TUG are assessed by a clinician, whereas ODI is assessed by the patient. Additionally, the ODI questionnaire focuses on activities of daily living rather than a timed test, which may result in differences in improvement. Patients may experience relief in different outcomes at different times, which is in line with other neuromodulation studies in that it often takes six months or longer to feel relief. ${ }^{14,15}$ Overall, results support the possibility of testing whether there is robust improvement in function and pain with HFIT treatment in a full-scale randomized controlled trial.

Although studies have shown that opioids can be effective in pain reduction, individual adverse events, such as nausea, vomiting, dizziness and constipation are more frequent in opioid users. ${ }^{16}$ In a meta-analysis of 22 randomized and quasi-randomized controlled trials that compared oral or transdermal opioids with no treatment in patients with hip or knee osteoarthritis, there was a difference in improvement of $12 \%$ between opioids and placebo, but patients receiving opioids had 3.76 times the risk of dropping out due to adverse events compared with patients who were not. ${ }^{17}$ Results from controlled clinical trials show approximately $50-70 \%$ of patients with chronic non-cancer pain fail opioid therapy and those who do respond report mild or moderate reduction in pain for a short term. ${ }^{18}$ When intermittently prescribed, opioids provide a very low-grade certainty of evidence when it comes to evaluating meaningful responses to treatment. Furthermore, opioids also come with the risk of addiction, misuse and overdose.

In comparison with TENS devices, HFIT also shows promising results. Although TENS has been widely used for over 30 years as a therapeutic complement to pain management, there is conflicting evidence in showing its effectiveness for CLBP. In a qualitative synthesis study, ${ }^{6}$ results from four placebo-controlled randomized controlled trials failed to consistently demonstrate whether TENS was beneficial in improving CLBP. Furthermore, a metaanalysis of twelve randomized TENS studies in treating CLBP suggested that TENS may offer short-term improvement of functional disability, but did not show improvement of LBP. ${ }^{19}$ Exercise therapy has been shown to provide a clinically meaningful reduction in pain and has also been one of the only interventions that demonstrates sustained improvement and relief. ${ }^{16}$ Because of the evidence behind the success of conservative therapies such as exercise, HFIT should not be a substitute for exercise therapy, but can instead be used to supplement and support such approaches. As a complement to exercise therapy, HFIT can work in conjunction by producing short-term pain relief which may allow patients to exercise more comfortably and minimize post-exercise pain. By using both therapies together, patients may be able to work towards achieving both short-term and long-term pain relief.

There are several inherent advantages to HFIT therapy. The therapy can be continued or discontinued quickly and does not require surgical intervention. Moreover, HFIT provides patients with a solution that does not limit function and physical therapy protocols, whereas treatments such as opioids can cause drowsiness and reduce overall function. Not only does HFIT avoid the potential adverse side effects, but it also provides local pain relief (without affecting any other parts of the body) that is both non-drug based and non-invasive.

Results from this study also demonstrated that device utilization was significantly higher in the active group throughout the course of the study, suggesting not only patients did experience a difference in pain relief when 

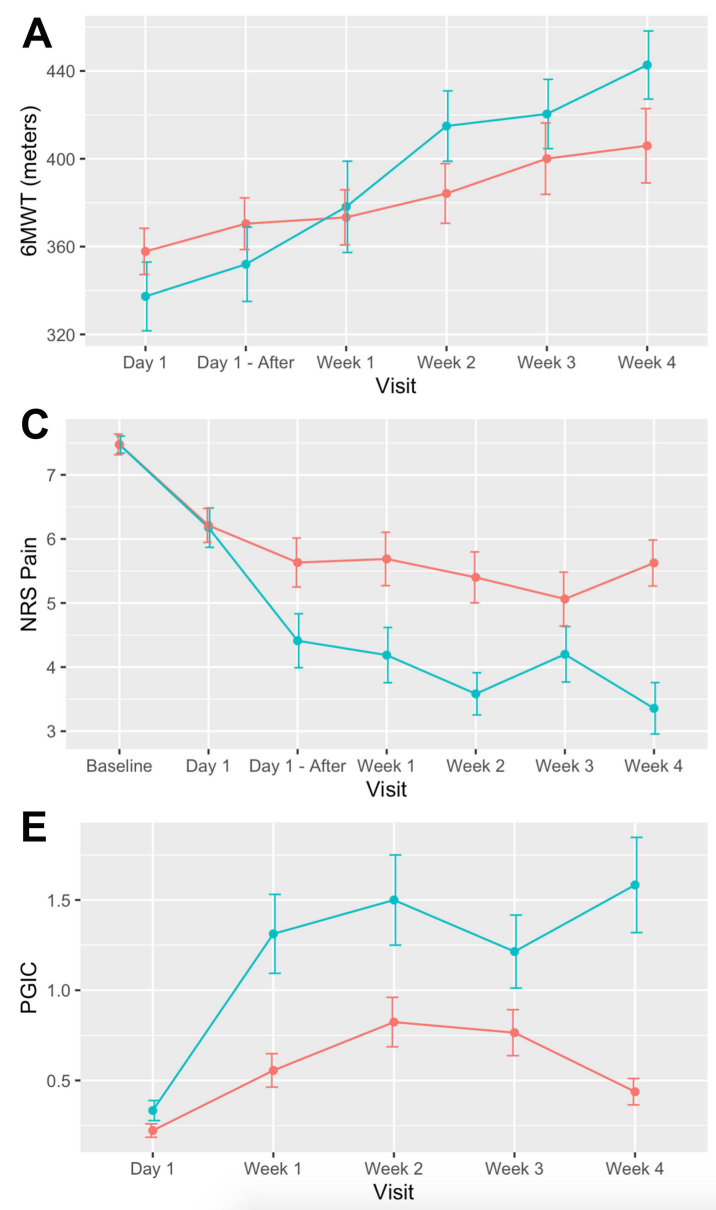
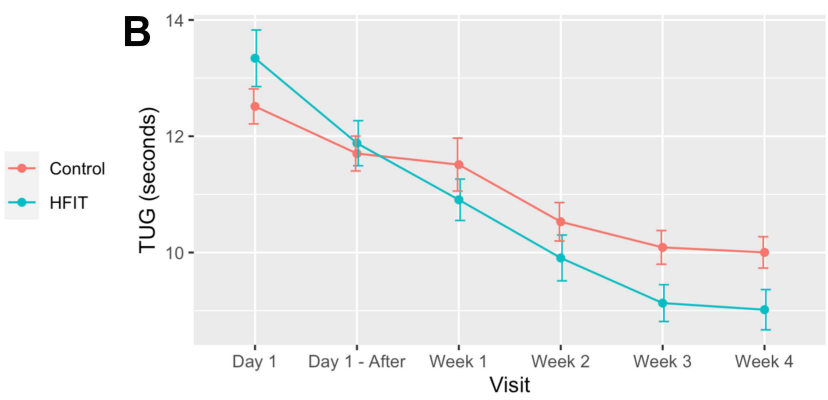

$\rightarrow$ Contro

$\rightarrow$ HFIT
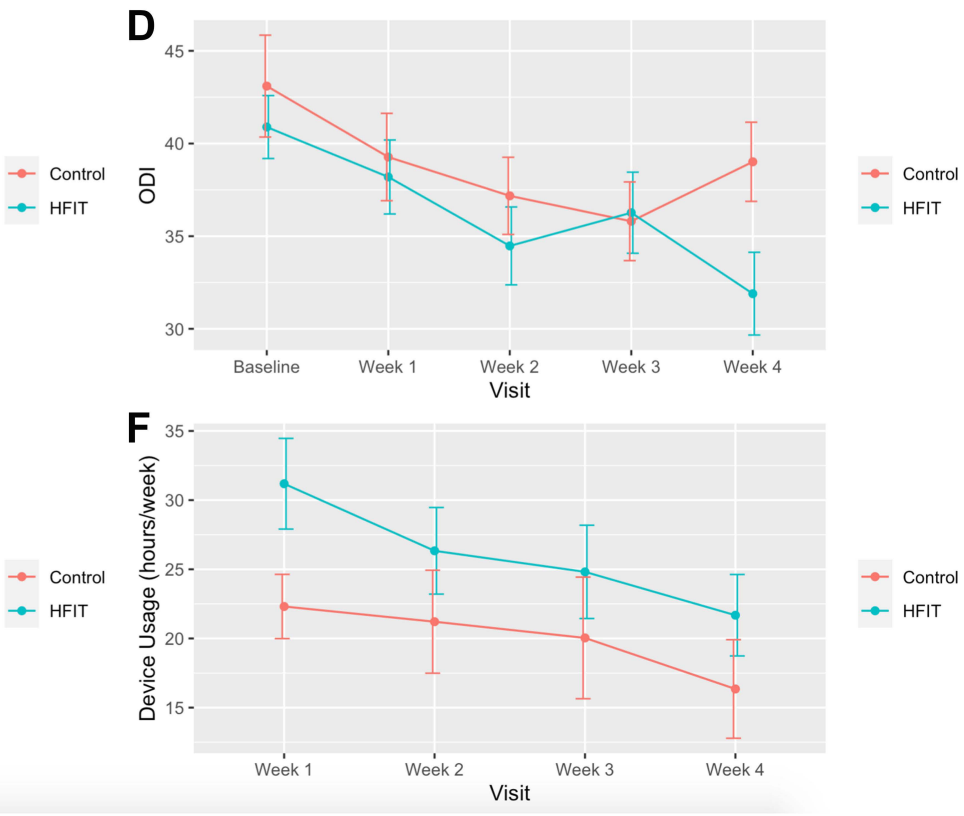

Figure 3 Longitudinal mean scores for outcomes.

Notes: (A) 6MWT, (B) TUG, (C) NRS Pain, (D) ODI, (E) PGIC, (F) Device Usage; Error bars show standard errors.

Abbreviations: 6MWT, Six Minute Walk Test; TUG, Timed Up and Go; NRS, Numerical Rating Scale; ODI, Oswestry Disability Index; PGIC, Patient Global Impression of Change.

using the HFIT therapy device but over time they preferred to continue using the device more regularly. While TENS units usually require direct adjustment of the hardware to modify the electrical stimulation parameters, the HFIT system is able to be modified using a communicating smartphone system. As a result, software adjustments may be performed remotely, which is distinct from current common TENS systems.

Strengths of this study include the study design, a multicenter double-blinded, sham-controlled randomized clinical trial. Strict methods were followed to assess the feasibility of conducting a full-scale trial. Multiple outcomes measuring function and pain were recorded. However, limitations of this study need to be acknowledged. Although the results presented support HFIT therapy as a viable treatment for chronic back pain, the data is from a pilot study with short-term follow-up results at 4 weeks and a full randomized controlled trial is required for more generalizable results. This pilot study adhered to strict inclusion and exclusion criteria, but enrollment and assessment numbers will be documented in future studies. This study also allowed for full patient control of their HFIT utilization. It is possible that users would expect a sensation from the HFIT device, even though they were informed they should not.

This pilot study shows encouraging early improvement in pain and functional assessments as well as increased utilization; however, future research using this pilot data is needed to advance HFIT as a mainstream treatment for CLBP. The results of this pilot study support conducting a larger, longerterm randomized controlled trial in order to evaluate sustained pain relief, improvement in function and reduction in analgesic use. Future studies will also develop protocols for use and location for treatment for various pain syndromes and other indications. 


\section{Conclusion}

This pilot study is the first evaluation of HFIT therapy for CLBP in a sham controlled, randomized manner. Patients who were randomized to use the HFIT device showed significant improvements in both functional and pain outcomes throughout the weeks of follow-up. Based on the results of this pilot trial, a larger-scale RCT is feasible and can build on the findings of this study to test whether HFIT is effective in reducing pain and improving function in CLBP patients. The efficacy, minimally invasive nature and ease of use of HFIT offer patients an alternative solution to pharmaceutical treatments and is anticipated to substantially improve the management of CLBP patients.

\section{Data Sharing Statement}

De-identified data are available upon request from the corresponding author.

\section{Acknowledgments}

We thank Shaun Rahimi, Grace Wang and Jeffrey Krauss for their helpful input into the study design, analysis and editing of this manuscript.

\section{Funding}

This research was funded by Thimble Bioelectronics Inc. dba Enso. At the time of the study, devices were donated by the manufacturer, Thimble Bioelectronics Inc. After the study was conducted, Thimble Bioelectronics was acquired by Hinge Health Inc.

\section{Disclosure}

Drs. Kasra Amirdelfan, Surekha Reddy, Vinay Reddy, Michael Yang and Amitabh Gulati received personal consulting fees from Thimble Bioelectronics Inc. Dr. Kasra Amirdelfan reports personal fees and/or advisory board from Nevro Inc, Medtronic, Boston Scientific, Saluda, Biotronik, Nalu, Presidio, Vivex, Mesoblast, and PainTEQ. Dr. Mindy Hong is employed at Hinge Health and received salary and nominal equity compensation. Dr. Bobby Tay reports personal fees for consulting from Biomet/Zimmer, Lumetra, Stryker, and DePuy Synthes Spine and institutional fellowship grants from OMEGA and NuVasive, outside the submitted work. Dr. Prasad Shirvalkar reports non-financial support from Abbott Neuromodulation, Nevro Inc, and Saluda Medical, outside the submitted work. In addition, Dr. Prasad Shirvalkar has a patent spinal cord stimulation methods pending. Dr. Amitabh Gulati is on the clinical advisory board of Hinge Health with nominal equity compensation. Dr. Amitabh Gulati also reports personal fees for consulting and/or advisory board from SPR Therapeutics, Nalu Medical, Medtronic Inc, AIS HealthCare, Tremeau Pharmaceuticals, and Flowonix, during the conduct of the study. The authors report no other potential conflicts of interest in this work.

\section{References}

1. Crow W, Willis D. Estimating cost of care for patients with acute low back pain: a retrospective review of patient records. J Am Osteopath Assoc. 2009;109(4):229-233.

2. Benyamin R, Trescot A, Datta S, et al. Opioid complications and side effects. Pain Physician. 2008;11(2):105-120. doi:10.36076/ppj.2008/ $11 / \mathrm{S} 105$

3. Abdel Shaheed C, Maher C, Williams K, Day R, McLachlan A. Efficacy, tolerability and dose-dependent effects of opioid analgesics for low back pain: a systematic review and meta-analysis. JAMA Intern Med. 2016;176(7):958. doi:10.1001/jamainternmed.2016.1251

4. Searle A, Spink M. Exercise interventions for the treatment of chronic low back pain: a systematic review and meta-analysis of randomised controlled trials. Clin Rehabil. 2014;29(12):1155-1167. doi:10.1177/0269215515570379

5. Deer T, Mekhail N, Provenzano D, et al. The appropriate use of neurostimulation of the spinal cord and peripheral nervous system for the treatment of chronic pain and ischemic diseases: the neuromodulation appropriateness consensus committee. Neuromodulation. 2014;17(6):515-550.

6. Khadilkar A, Odebiyi D, Brosseau L, Wells G. Transcutaneous electrical nerve stimulation (TENS) versus placebo for chronic low-back pain. Cochrane Database Syst Rev. 2008. doi:10.1002/14651858. CD003008.pub3

7. Hughes N, Bennett MI, Johnson MI. An investigation into the magnitude of the current window and perception of transcutaneous electrical nerve stimulation (TENS) sensation at various frequencies and body sites in healthy human participants. Clin J Pain. 2013;29 (2):146-153. doi:10.1097/AJP.0b013e3182579919

8. Facci LM, Nowotny JP, Tormem F, Trevisani V. Effects of transcutaneous electrical nerve stimulation (TENS) and interferential currents (IFC) in patients with nonspecific chronic low back pain: randomized clinical trial. Sao Paulo Med J. 2011;129(4):206-216. doi:10.1590/S1516-31802011000400003

9. Kapural L, Yu C, Doust MW, Gliner BE, Vallejo R. Novel 10-kHz high-frequency therapy (HF10 therapy) is superior to traditional low-frequency spinal cord stimulation for the treatment of chronic back and leg pain: the SENZA-RCT randomized controlled trial. Anesthesiology. 2015;123(4):851-860. doi:10.1097/ALN.0000000 000000774

10. Moran F, Leonard T, Hawthorne S, et al. Hypoalgesia in response to transcutaneous electrical nerve stimulation (TENS) depends on stimulation intensity. J Pain. 2011;12(8):929-935. doi:10.1016/j. jpain.2011.02.352

11. Beebe SJ. Bioelectrics in basic science and medicine: impact of electric fields on cellular structures and functions. $J$ Nanomed Nanotechnol. 2013;4(2). doi:10.4172/2157-7439.1000163

12. Whitehead AL, Julious SA, Cooper CL, Campbell MJ. Estimating the sample size for a pilot randomised trial to minimise the overall trial sample size for the external pilot and main trial for a continuous outcome variable. Stat Methods Med Res. 2016;25(3):1057-1073. doi:10.1177/0962280215588241 
13. Hertzog MA. Considerations in determining sample size for pilot studies. Res Nurs Health. 2008;31(2):180-191. doi:10.1002/ nur.20247

14. Staelin R, Koneru S, Rawe IM. A prospective six-month study of chronic pain sufferers: a novel OTC neuromodulation therapy. Pain Res Manag. 2019;2019(3):1-11. doi:10.1155/2019/3154194

15. Petersen EA, Stauss TG, Scowcroft JA. Effect of high frequency $(10-\mathrm{kHz})$ spinal cord stimulation in patients with painful diabetic neuropathy. JAMA Neurol. 2021;78(6):687-698. doi:10.1001/ jamaneurol.2021.0538

16. Kolber M, Ton J, Thomas B, et al. PEER systematic review of randomized controlled trials. Can Fam Physician. 2021;67(1):20-30.
17. Costa B, Nüesch E, Kasteler R, et al. Oral or transdermal opioids for osteoarthritis of the knee or hip. Cochrane Database Syst Rev. 2014;17(9).

18. Sehgal N, Colson J, Smith HS. Chronic pain treatment with opioid analgesics: benefits versus harms of long-term therapy. Expert Rev Neurother. 2013;13(11):1201-1220. doi:10.1586/14737175.20 13.846517

19. Wu L, Weng P, Chen C, et al. Literature review and meta-analysis of transcutaneous electrical nerve stimulation in treating chronic back pain. Reg Anesth Pain Med. 2018;43(4):425-433. doi:10.1097/ AAP.0000000000000740

\section{Publish your work in this journal}

The Journal of Pain Research is an international, peer reviewed, open access, online journal that welcomes laboratory and clinical findings in the fields of pain research and the prevention and management of pain. Original research, reviews, symposium reports, hypothesis formation and commentaries are all considered for publication. The manuscript

Submit your manuscript here: https://www.dovepress.com/journal-of-pain-research-journal management system is completely online and includes a very quick and fair peer-review system, which is all easy to use. Visit http:// www.dovepress.com/testimonials.php to read real quotes from published authors. 